OAI-PMH: http://www.indteca.com/ojs/index.php/Revista Scientific/oai

Artículo Original / Original Article

\title{
El Sentido social del aprendizaje por experiencia en los discursos legales y universitarios venezolano
}

\author{
Autora: Maeva Elena Hernández Pereira \\ Universidad Nacional Experimental Politécnica de la Fuerza Armada, UNEFA \\ maevaunefa2012@gmail.com \\ Caracas, Venezuela \\ https://orcid.org/0000-0001-5563-4930
}

\section{Resumen}

La presente investigación responde a interrogantes: ¿qué sentido social le ha asignado el venezolano a los aprendizajes adquirido por experiencia?; ¿qué significados son asignados en los discursos legales y universitarios?; ¿qué representaciones sociales emergen en esa interdiscursividad?; ¿qué fundamentos teóricos sustentarían dichas representaciones simbólica 0 sentido social?. Determino el sentido social que el venezolano ha asignado al aprendizaje por experiencia, específicamente en los discursos legales y universitarios. Utilizando la teoría sociosemiótica estructuralista (hermenéutica intertextual) acompañada de un análisis interdiscursivo documental de tipo legal (leyes, decretos y resoluciones de la nación) y universitario (normas, reglamento, programas académicos) y de la codificación selectiva para las categorías de sentido. El principal hallazgo del significado legal representando, se refiere al "enlace generacional" y el significado universitario con forma de "herramienta flexibilizadora". Entre los resultados se destacan las representaciones simbólicas del conocimiento como bizarro, demostración; resolutivo, contraste y subjetivo. Concluyéndose que dicho conocimiento hoy día simboliza la identidad subjetiva de la persona en su momento histórico, quedando el desafío para las universidades de investigar cómo orientar los procesos de reconocimiento, validación y acreditación de dicho aprendizaje.

Palabras clave: sentido social; semiótica; representaciones del conocimiento; discurso.

Código de clasificación internacional: 5705.10 - Sociolingüística.

Cómo citar este artículo:

Hernández, M. (2021). El Sentido social del aprendizaje por experiencia en los discursos legales y universitarios venezolano. Revista Scientific, 6(20), 171-189, e-ISSN: 2542-2987. Recuperado de: https://doi.org/10.29394/Scientific.issn.2542-2987.2021.6.20.9.171-189

Fecha de Recepción: 05-08-2020
Fecha de Aceptación: 09-02-2021
Fecha de Publicación: 05-05-2021 
OAl-PMH: http://www.indteca.com/ojs/index.php/Revista Scientific/oai

Artículo Original / Original Article

\title{
The social sense of experience learning in Venezuelan legal and university discourses
}

\begin{abstract}
This research responds to questions: what social meaning has the Venezuelan assigned to the learning acquired by experience?; What meanings are assigned in legal and university discourses ?; What social representations emerge in this interdiscursiveness?; What theoretical foundations would sustain these symbolic representations or social meaning? I determine the social meaning that the Venezuelan has assigned to learning by experience, specifically in legal and university speeches. Using the structuralist sociosemiotic theory (intertextual hermeneutics) accompanied by a documentary interdiscursive analysis of a legal type (laws, decrees and resolutions of the nation) and university (norms, regulations, academic programs) and of the selective codification for the categories of meaning. The main finding of the legal meaning representing refers to the "generational link" and the university meaning in the form of a "flexibilizing tool". Among the results stand out the symbolic representations of knowledge such as bizarre, demonstration; resolutive, contrast and subjective. Concluding that said knowledge today symbolizes the subjective identity of the person in their historical moment, leaving the challenge for universities to investigate how to guide the processes of recognition, validation and accreditation of said learning.
\end{abstract}

Keywords: social sense; semiotics; representations of knowledge; speech.

International classification code: 5705.10 - Sociolinguistics.

\footnotetext{
How to cite this article:

Hernández, M. (2021). The social sense of experience learning in Venezuelan legal and university discourses. Revista Scientific, 6(20), 171-189, e-ISSN: 2542-2987. Recovered from: https://doi.org/10.29394/Scientific.issn.2542-2987.2021.6.20.9.171-189
}

Date Received: 05-08-2020
Date Acceptance: 09-02-2021
Date Publication: 05-05-2021 


\section{Introducción}

El concepto asignado al aprendizaje adquirido por experiencia, también denominado aprendizaje informal, o aprendizaje no convencional, el cual, es tema de debate y actualmente se coloca con renovado interés en el tapete de la discusión.

En América Latina durante el siglo XIX, de acuerdo con el Centro Interamericano para el Desarrollo del Conocimiento en la Formación Profesional y la Organización Internacional del Trabajo (Cinterfor/OIT, 1990): hablar de aprendizaje por experiencias remitía a la formación no formal; o informal de instituciones ubicada fuera del sistema educativo. Tales instituciones fueron las encargadas de la formación de mano de obra calificada, o la formación de recurso humano para sustentar el proceso de industrialización y sustitución de importaciones; ya fuese con financiamiento estatal o privado.

Para el siglo XX la fuente del aprendizaje se encontraba polarizada; por un lado, los ministerios de educación, quienes asumían la formación técnicaprofesional mediante las escuelas de artes y oficios para el desarrollo industrial; por otro lado, el sector empresarial colaboraba con el estado, específicamente con los ministerios del trabajo, en la contratación y formación de los jóvenes aprendices en los oficios laborales.

En el siglo XXI, Delors (1996): advierte que predominará la educación a lo largo de toda la vida. Ello conllevará a una educación pludimensional constituida por formación técnica, profesional y competencias adquiridas durante la experiencia vivida cotidianamente, conforme a las necesidades del lugar. El Instituto de la Organización de las Naciones Unidas para la Educación, la Ciencia y la Cultura (UNESCO, 2012a): define que "el aprendizaje a lo largo de toda la vida va más allá de la educación y la capacitación formal. Incluye el aprendizaje en el trabajo, en la familia y la comunidad, así como durante el tiempo de ocio" (pág. 4). 
Esto significa la apertura a nuevas oportunidades para el aprendizaje no convencional y posibilita que la personas se beneficien independientemente de sus circunstancias. Vale destacar que la Unesco, le asigna un elemento más en este significado al preferir aplicarlo preferentemente a personas desfavorecidas que requieren de equidad de acceso al sistema educativo. Ponderando con ello: el carácter del entorno, la circunstancia; el contexto, lo resolutivo y práctico del significado asignado. La Organización de las Naciones Unidas para la Educación, la Ciencia y la Cultura (UNESCO, 2012b), agrega a dicho aprendizaje que el:

Reconocimiento: es un proceso que asigna un estatus oficial a los resultados o competencias del aprendizaje, lo que puede conducir al reconocimiento de su valor en la sociedad; Validación: es la confirmación por un órgano autorizado [...]; Acreditación: es un proceso mediante el cual un órgano autorizado [...] asigna calificaciones (certificados, diplomas o títulos) u otorga equivalencias, unidades de crédito o excepciones, o emite documentos tales como portafolios de competencias (pág. 8).

Así el aprendizaje pasa de ser un fin teórico abstracto de información y conocimiento para el desarrollo del individuo, a ser un medio práctico resolutivo contextualizado; generador de resultados positivos en diversas situaciones y un aprendizaje de calidad. Este último, precisado por la Organización Internacional del Trabajo y el Centro Interamericano para el Desarrollo del Conocimiento en la Formación Profesional (OIT/Cinterfor, 2017), definen el aprendizaje de calidad:

Como una forma única de formación profesional, que combina la formación en el puesto de trabajo y el aprendizaje basado en el aula, para el desarrollo de competencias y procesos de trabajo específicamente definidos [...] con una evaluación formal y certificación final de las habilidades y competencias adquiridas (pág. 77).

Aquí el significado de dicho aprendizaje es ser "bizarro" mediadora entre 
sistema educativo y el sector laboral; siempre y cuando, demuestre su utilidad. Mientras que para la Organización de las Naciones Unidas para la Educación, la Ciencia y la Cultura (UNESCO, 2017): significaba patrimonio cultural de las comunidades y las habilidades pertinentes necesarias en determinados entornos, sea laboral comunitario.

En este contexto evolutivo de los significados cabe preguntarse: ¿qué sentido social asigna el venezolano a los aprendizajes adquiridos por experiencia?; ¿qué significados atribuye a dicho aprendizaje en los discursos legales y universitarios?; ¿qué representaciones sociales emergen en esa interdiscursividad?; ¿qué fundamentos teóricos sustentarían dichas representaciones o sentido social asignado a dicho aprendizaje en los discursos señalados?.

Para lograrlo, esta investigación propone determinar el sentido social asignado por el venezolano a los aprendizajes adquiridos por experiencia, identificar los significados de aprendizaje por experiencia existente en los discursos legales y universitarios en Venezuela, interpretar las representaciones sociales sobre el aprendizaje existentes en la interdiscursividad académica y fundamentar teóricamente los sentidos sociales encontrados. La importancia de alcanzar dichos objetivos radica en que ellos permitirían comprender la importancia de su reconocimiento y legitimización, en la formación de por vida del ciudadano.

\subsection{Referencias teóricas}

Es necesario recordar a Verón (1993a): quien define la discursividad social o teoría de la producción de sentido, al igual que "[...] la semiosis donde se construye la realidad de lo social [...] al estudio de la construcción social de lo real [...] la parte ideal de lo real [...] sin embrollarse con un modelo subjetivista del actor" (pág. 126).

El sentido social es definido por representaciones simbólicas 
entrelazadas con los comportamientos sociales en espacios y tiempos determinados que construyen la realidad social. En ese marco, Verón (1993b): explica que la representación social, son modelos socio cognitivos contextualizados; organizados en dispositivos discursivos, con los cuales el ser humano interpreta sus interacciones con otros y con el mundo social en el que se encuentra.

Tales discursos están constituidos, según Verón (2004a): por una gramática de producción que representa "[...] un campo de efectos de sentido posibles [...]" (pág. 130); la misma contiene operadores, entendidos estos como marcas discursivas, cuando representan condiciones sociales del momento histórico en el cual se desarrollan los discursos en referencia; y entendidos como operadores huellas discursivas cuando visibilizan la relación del significado con la condición de producción o reconocimiento del discurso.

Acorde con esto, para Verón (2004b): “[...] la gramática de reconocimiento aplicada a un texto en un momento dado, sigue siendo insoluble a la sola luz de las reglas de producción: sólo puede resolverse en relación con la historia de los textos" (pág. 130); es decir, aquella que se visibiliza en las materias de significantes de la sociedad en referencia a lo largo de su historia.

\section{Metodología}

Para analizar tales gramáticas discursivas se requerirá de al menos dos corpus discursivos: a). Discursos legales llamados discursos de producción: aquellos generadores de efectos e impactos en otros discursos, utilizándose para su selección la alta frecuencia de citas realizadas por las universidades venezolanas, cada vez que se aborda el tema del aprendizaje por experiencia; b). Discursos universitarios llamados de reconocimiento: aquellos que reflejan en sus gramáticas huellas y marcas de discursos legales, del mismo modo reglamentos, programas, resoluciones de consejos universitarios, entre otros. 
Cuya selección respondería a su frecuencia de citas por la comunidad universitaria

De esta forma, se utilizó el análisis interdiscursivo propuesto por Verón (2004b): para visibilizar la multiplicidad de sentidos que se producen en la red semiótica, entendida ésta en el intercambio de discursos en donde los actores sociales asignan sentidos a la realidad social vivida. No existiendo entre dichos discursos, causalidad lineal sino multiplicidad de efectos posibles.

Acompañado de técnicas de formulación de preguntas sensibilizadoras, determinadas por Strauss y Corbin (2002a): aquellas que "ponen al investigador en sintonía con lo que los datos [...]" (pág. 84); comparaciones entre gramática discursiva: empleada para identificar marcas y huellas, para lo que Verón (1993c): se manifiesta en los intercambios discursivos de efectos de un discurso sobre otros.

Para lograrlo se aplicarán los siguientes procedimientos: a). Identificación de las gramáticas de cada discurso, seleccionado en los corpus mencionados; b). Aplicación de preguntas sensibilizadoras, ¿cómo cada discurso define el aprendizaje por experiencia?; ¿qué significado le asignan?.

En cada una de las gramáticas discursivas seleccionadas de cada discurso: ¿son sus significados los mismos o diferentes?; c). Agrupación de dichas respuestas en categorías, especificadas por Strauss y Corbin (2002b): de la siguiente manera "[...] conceptos que representan fenómenos [...]" (pág. 110); y "[...] términos explicativos más abstractos [...]" (págs. 125); d). Interrelacionamiento, entre sí, de cada categoría a fin de redactar una explicación precisa y completa sobre el fenómeno en estudio, proceso llamado por Strauss y Corbin (2002c): la "Codificación axial" (pág. 134); e). Fundamentación teórica de los sentidos sociales encontrados en dichos discursos, seleccionándose el aporte teórico según el tema central del sentido social encontrado.

En la validación de los resultados se aplicarán los criterios y técnicas propuestas por Franklin y Ballau (2005), citados por Hernández, Fernández y 
Baptista (2010): a. Grado de coincidencia de los datos en fuentes distintas a lo legal e institucional; b. Grado de corroboración estructural, es decir, el "[...] proceso mediante el cual varias partes de los datos (categorías, por ejemplo), se "soportan conceptualmente" entre sí (mutuamente)" (pág. 475); y 3). Triangulación teórica para "[...] analizar los mismos datos bajo diferentes visiones teóricas o campos de estudio" (pág. 476).

\section{Resultados}

En el cuadro 1, se muestran los dieciséis (16) discursos legales venezolanos representativos del significado social asignado al aprendizaje por experiencia, para el período histórico comprendido entre el año 1883 al 2014, observándose que desde el año 1880 hasta 1930, el aprendizaje por vía de experiencia significaba en los discursos legales una habilidad adquirida en la práctica cotidiana por vía individual o familiar. Entre 1930 hasta 1975 paso a significar una condición necesaria para calificar en un oficio laboral o para acceder a un aprendizaje institucionalizado. Para el lapso comprendido entre 1975 a 1995 el aprendizaje por experiencia representaba un conjunto de habilidades y destrezas que caracterizaban a una persona sin formación académica.

Entre tanto, a partir de los años 1995 al 2003, en los instrumentos legales venezolanos, significaba la capacidad que tenía una persona para solucionar algún problema o satisfacer una determinada necesidad fuese esta: laboral, comunitaria, familiar, individual, etc. Comenzando con el año 2003 y hasta el 2012, el significado del aprendizaje se desprende del sujeto portador del mismo y pasa a significar un mecanismo para: validar, diferenciadamente los aprendizajes académicos, de los otros.

Esto conlleva a las instituciones formativas a flexibilizar el sistema educativo venezolano por vía de comparación, equivalencia, reconocimientos u otras modalidades. En el año 2012 y hasta la actualidad es un derecho del 
sujeto portador de que dicho aprendizaje por experiencia sea reconocido académicamente. Pasando a ser un deber de las academias, ajustar sus protocolos administrativos para legitimar dicho derecho.

Cuadro 1. Significado social del aprendizaje adquirido por experiencia en los discursos legales venezolanos.

\begin{tabular}{|c|c|c|}
\hline Año & Documento Legal & $\begin{array}{c}\text { Significado Social del Aprendizaje Adquirido } \\
\text { por experiencia }\end{array}$ \\
\hline 1883 & $\begin{array}{l}\text { Decreto } 24 \text { septiembre por Antonio } \\
\text { Guzmán Blanco. }\end{array}$ & Habilitación de experiencia. \\
\hline 1897 & Código Instrucción Pública. & $\begin{array}{l}\text { Legitimación del saber empírico instrumental } \\
\text { individual y familiar. }\end{array}$ \\
\hline 1936 & Ley del Trabajo. & Insumo para cualificar. \\
\hline 1954 & $\begin{array}{l}\text { Ley del Instituto para la Capacitación y } \\
\text { Recreación de los Trabajadores } \\
\text { (INCRET). }\end{array}$ & Mejorar lo aprendido en el Oficio. \\
\hline 1959 & $\begin{array}{l}\text { Ley de creación del Instituto Nacional de } \\
\text { Cooperación Educativa (INCE). }\end{array}$ & Formación laboral especializada. \\
\hline 1962 & $\begin{array}{l}\text { Programa de Capacitación Laboral } \\
\text { Juvenil del INCE }\end{array}$ & Insumo para diagnóstica cualificación laboral. \\
\hline 1970 & Ley Universidades. & $\begin{array}{l}\text { Características del Ente } \\
\text { aprendizaje. }\end{array}$ \\
\hline 1980 & Ley Orgánica de Educación. & Características del Ente Portador. \\
\hline 1996 & Ministerio de Educación. & Demostración del saber hacer. \\
\hline 1999 & $\begin{array}{l}\text { Reglamento General de la Ley Orgánica } \\
\text { de Educación. }\end{array}$ & Satisfacción de necesidades concretas. \\
\hline 1999 & Constitución de la República. & Satisfacción demanda laboral. \\
\hline 2003 & $\begin{array}{l}\text { Ministerio de Educación (Resolución N. } \\
65 \text { ). }\end{array}$ & $\begin{array}{l}\text { Cantidad de Unidades Curriculares equivalente a } \\
\text { competencias adquiridas. }\end{array}$ \\
\hline 2008 & Ley del INCES & $\begin{array}{l}\text { Demanda flexibilización del Sistema Educativo. } \\
\text { Demostración de problemas y necesidades } \\
\text { satisfechas con las competencias a acreditar. }\end{array}$ \\
\hline 2009 & Ley Orgánica de Educación. & $\begin{array}{l}\text { Legitimación institucional del aprendizaje } \\
\text { adquirido por experiencia y la autoformación. }\end{array}$ \\
\hline 2011 & 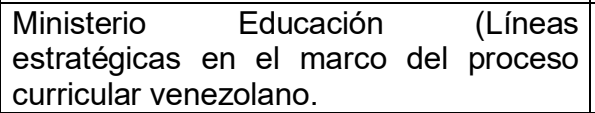 & $\begin{array}{l}\text { Reconoce aprendizaje informales } \\
\text { autoformación. }\end{array}$ \\
\hline 2014 & Reforma de la Ley del INCES & Flexibilización del Protagonista. \\
\hline
\end{tabular}

Fuente: La Autora (2021).

El cuadro 2, muestra once (11) discursos universitarios de reconocimiento de los discursos legales; representativos del significado asignado al aprendizaje adquirido por vía de experiencia en el lapso comprendido para el año 1980 al 2016. Observándose en primera instancia 
que es hasta finales de la década de los ochenta cuando en los discursos de las universidades venezolanas comienza a tener algún significado el aprendizaje por experiencia. Entendiéndose éste como un acto de presión ejercido por el entorno externo, no universitario, obligando a la academia a incorporar protocolos para incluir a dicha población.

Dicha tendencia inclusiva predomina en toda la década de los noventa; incorporándose las características socioeconómicas y financieras del sujeto en condición para el acceso a la formación universitaria. Es apenas en el período comprendido entre el año 2000 al 2010, en donde se reconoce dicho aprendizaje y conocimiento resolutivo que podría ser sometido a validación por los sectores productivos y empresariales; a fin de ser reconocido por la academia. Es de aclarar, que significaba un saber empírico instrumental, que mediaba lo laboral y lo académico.

A inicios del año 2010 al 2015 el aprendizaje por experiencia en Venezuela pasa a ser reconocido como: conocimiento que requiere de un proceso de validación, reconocimiento y acreditación, en relación con algunas unidades curriculares existentes, en el pensum curricular de las carreras aspiradas por él o los sujeto(s) portador(es) de la experiencia.

Hace cinco años la mediación laboral/académica (o los procesos académicos protocolares), han venido cediendo espacios para emerger el sujeto portador del aprendizaje por experiencia, protagonista de su propio diseño de pensum curricular.

Cuadro 2. Significado social del aprendizaje adquirido por experiencia en discursos universitarios venezolanos.

\begin{tabular}{|c|l|l|}
\hline Año & \multicolumn{1}{|c|}{ Universidad / Documento } & \multicolumn{1}{c|}{$\begin{array}{c}\text { Significado Social del Aprendizaje Adquirido } \\
\text { por experiencia }\end{array}$} \\
\hline \multirow{3}{*}{1981} & $\begin{array}{l}\text { Universidad Nacional Experimental } \\
\text { Simón Rodríguez (UNESR): } \\
\text { Acreditación del Aprendizaje por } \\
\text { Experiencia (PAAPE). }\end{array}$ & $\begin{array}{l}\text { Accesibilidad de los excluidos al Sistema } \\
\text { Educativo. } \\
\text { Dignificación educativa de los resultados de la } \\
\text { experiencia. }\end{array}$ \\
\hline
\end{tabular}


Instituto Internacional de Investigación y Desarrollo Tecnológico Educativo INDTEC, C.A.

DOI: https://doi.org/10.29394/Scientific.issn.2542-2987.2021.6.20.9.171-189

OAI-PMH: http://www.indteca.com/ojs/index.php/Revista Scientific/oai

Artículo Original / Original Article

\begin{tabular}{|c|c|c|}
\hline 1988 & $\begin{array}{l}\text { Universidad Nacional Abierta (UNA): } \\
\text { Normativa de evaluación (Resolución } \\
\text { S-2- 379, UNA, CD, 1988). }\end{array}$ & $\begin{array}{l}\text { Enfatiza el proceso de acreditación y sus } \\
\text { procedimientos. }\end{array}$ \\
\hline \multirow{2}{*}{$\begin{array}{l}\text { Década } \\
\text { de los } 90\end{array}$} & Programas de Extensión Universitaria. & $\begin{array}{l}\text { Formación ajustada a características y } \\
\text { situaciones de la población no vinculada a } \\
\text { programas de pregrado y postgrado. }\end{array}$ \\
\hline & $\begin{array}{l}\text { Institutos de Formación Laboral } \\
\text { adscrito al Ministerio del Trabajo. }\end{array}$ & $\begin{array}{l}\text { Formación laboral legitima aprendizaje adquirido } \\
\text { por experiencia. }\end{array}$ \\
\hline 2005 & $\begin{array}{l}\text { Universidad Nacional Experimental de } \\
\text { los Llanos Occidentales Ezequiel } \\
\text { Zamora (UNELLEZ): Normas para la } \\
\text { Acreditación del Aprendizaje por } \\
\text { Experiencia (Resolución del Consejo } \\
\text { Directivo: } 2005 / 531 \text { de fecha } 26 / 07 / 05 \text {. } \\
\text { Punto 49). }\end{array}$ & $\begin{array}{l}\text { Valoración educativa del saber práctico } \\
\text { adquirido por fuentes y métodos no tradicionales } \\
\text { del Sistema Educativo Venezolano. }\end{array}$ \\
\hline 2007 & $\begin{array}{l}\text { Universidad Nacional Experimental de } \\
\text { Guayana (UNEG): Reglamento para la } \\
\text { Acreditación de Aprendizaje por } \\
\text { Experiencia (Resolución N. }{ }^{\circ} \text { CU-0-12- } \\
\left.563 \text { Acta N. } .^{\circ} \mathrm{O}-12\right) \text {. }\end{array}$ & $\begin{array}{l}\text { Aprendizajes relevantes para la carrera aspirada } \\
\text { por el solicitante. }\end{array}$ \\
\hline 2008 & $\begin{array}{l}\text { Universidad Bolivariana de Venezuela } \\
\text { (UBV): Programas Nacionales de } \\
\text { Formación - PNF en educación. }\end{array}$ & $\begin{array}{l}\text { Flexibilización Curricular Acreditación de } \\
\text { competencias resolutivas en contexto. }\end{array}$ \\
\hline 2011 & $\begin{array}{l}\text { Universidad Nacional Experimental de } \\
\text { la Seguridad (UNES): Reglamento de } \\
\text { Organización y Funcionamiento de la } \\
\text { UNES (Decreto N. } .^{\circ} 8.014, \text { Gaceta } \\
\text { Oficial N. }{ }^{\circ} 39.602 \text { ). }\end{array}$ & $\begin{array}{l}\text { Forma parte del pensum curricular como una } \\
\text { unidad curricular del PNF. }\end{array}$ \\
\hline 2012 & $\begin{array}{l}\text { Gobierno Nacional crea Universidades } \\
\text { Politécnicas Territoriales (Gaceta } \\
\text { Oficial N. }{ }^{\circ} \text { 39.902). }\end{array}$ & $\begin{array}{l}\text { Dialogo horizontal entre contenidos académicos } \\
\text { y contenidos derivado de experiencias en } \\
\text { espacio, tiempo, lugar y sujetos específicos. }\end{array}$ \\
\hline 2016 & $\begin{array}{l}\text { Universidad Politécnica Territorial de } \\
\text { Mérida Kléber Ramirez (UPTM): } \\
\text { Reglamento para la Presentación del } \\
\text { Trabajo de Investigación para optar al } \\
\text { Grado de Especialista y Magister } \\
\text { Scientiae (Gaceta Oficial N. }{ }^{\circ} \text { 41.056). }\end{array}$ & $\begin{array}{l}\text { Aspirantes reconocen sus propios aprendizajes } \\
\text { formales y no formales y diseñan su propia } \\
\text { agenda universitaria. }\end{array}$ \\
\hline
\end{tabular}

Fuente: La Autora (2021).

\subsection{Interpretación y discusión de los resultados}

En la identificación, comprensión, e interpretación de los impactos o influencia que los discursos legales dejaron en los discursos universitarios; se logra determinar el sentido social asignado al aprendizaje adquirido por experiencia. 


\subsubsection{Sentido Social Bizarro}

Entre los años 1883 hasta 1995 los discursos legales exigían, a las instituciones venezolanas, que valorizaran dicho aprendizaje, no obstante, estas exigencias respondían con modalidades formativas con poca exigencia académica, orientado más a la inclusión y acceso de dicha población al sistema. Asignando a dicho conocimiento el significado de bizarro entre el saber popular cotidiano y el conocimiento académico de bajo perfil y exigencia.

Se considera a su portador: un manipulador de herramientas prácticas; legitimadas por el adiestramiento, capacitación y actualización del Oficio Laboral. Equivale también a una estrategia relacional para articular la academia con el mundo laboral, manteniendo la academia en convivencia con la cultura y el tejido de la sociedad; en la cual se encuentra inmersa buscando mutuo beneficio.

En la fundamentación teórica de dicho conocimiento se encuentran los aportes de Hopenhayn y Ottone (2000): a quienes les caracteriza por reducir las desigualdades de cara al futuro y superar la reproducción intergeneracional de la pobreza, constructor de ciudadanía, en la medida que intenta perfeccionar la institucionalidad académica y un mediador entre la cultura popular y el sistema educativo.

Destacando para dichos autores este tipo de conocimiento se valoraría por su grado de subordinación a la relación medio-fin, para organizar los aprendizajes y aplicarlo. De ese modo, la Universidad sería la mediadora entre el conocimiento y el Ser; quien reconoce: su saber hacer, potencialidades, y derechos. El aprendizaje se presenta a manera oportunidad institucional para humanizar el conocimiento, centrándolo en la persona; sus emociones y cogniciones, reconociendo la motivación.

\subsubsection{Sentido social Demostrativo}

Entre los años 1996 al 2002, la interacción entre los discursos legales y 
los universitarios, generan la representación social de conocimiento; no estructurado que requería de tratamiento especializado para demostrar su validez. Representa un saber-conocer, es decir, dominio conceptual y práctico de conocimientos procedimentales; operativos de acción circunstancial o por objetivos concretos; significaba: saber aplicado.

Exigía el aporte de evidencia sobre: lo que se sabe y lo que puede demostrarse de eso que se sabe. Mediante este proceso, señala Duval (1999), citado por Valiente (2000): se activa el aprendizaje razonado debido a que demostrar significa organizar y explicar, con argumentación, la justificación de las propiedades, virtudes de aquello que se desea demostrar.

Durante el proceso de demostración, lo ha destacado Villiers (1993): en el sujeto portador que auto explica la forma, en el cual lo adquirió y la utilidad de este. Siendo uno de sus principales componentes, la deducción, reconstrucción y la argumentación, razones encadenadas lógicamente a hechos concretos que ratifican determinadas afirmaciones.

\subsubsection{Sentido social resolutivo}

El sentido social resolutivo es: lo que para el venezolano significaba el aprendizaje por experiencia entre los años 2003 al 2008; un saber o conocimiento, orientado a la solución de problemas y la satisfacción de necesidades concretas. Su portador es: un Ser solucionador lleno de creatividad e innovación; consciente de la forma de movilizar sus recursos cognitivos, con pensamiento crítico y compromiso social.

En este aspecto, Dewey (1998): considera este tipo de conocimiento, seguido de la asignación de sentido en contexto, debido a que su aplicación y resultado permite darle significado a la experiencia vivida. Sobre eso, Torp y Sage (2007a): lo consideraban solución al "[...] centro organizador y contexto para el aprendizaje [...]" (pág. 49); durante el proceso.

La solución a dicha problemática, resumiendo los aportes de Torp y 
Sage (2007b): promueve en el Sujeto, la motivación, debido al interés de buscar solución, significación debido a que permite asignar sentido a lo vivido, pensamiento crítico-creativo debido a que se busca la respuesta correctaeficiente, metacognición y autorregulación debido a que genera estrategias para encontrar el problema, reunir información, analizar datos, construir hipótesis; ponerlas a prueba, evaluar sus resultados y compararlos con otros.

\subsubsection{Sentido social por contraste de perfiles}

El sentido social por contraste de perfiles emerge aproximadamente en el período 2009 al 2011, interpretándose aquel conjunto de competencias en la que el sujeto adquiere consciencia de poseerla, después de haber sido sometido a un proceso de comparación entre su Ser real, con el perfil de un Ser teórico legitimado institucionalmente. Entendiéndose Perfil profesional, a juzgar por Arnaz (2016): es el conjunto de experiencias educativas y características que posee un sujeto. En donde se diagnosticaban, en el sujeto portador, rasgos cognitivos, afectivos y fisiológicos, que sirvieran de indicadores para la toma de decisión sobre el acceso a la carrera solicitada por el sujeto portador.

Representa dicho conocimiento un proceso de: revivir lo vivido, sistematizando y extrayendo de ello competencias adquiridas. El sujeto portador comprendió su importancia en el desempeño laboral, ello requiere de actitud de alerta y dedicación sostenida, acorde con esto, para Páez y Puig (2013): solo así el sujeto portador sería capaz de problematizar lo vivido, interrogarlo y extraer acciones y emociones aprendidas.

\subsubsection{Sentido social, reflexión-subjetiva de lo vivido}

Aproximadamente en el año 2012, el aprendizaje por experiencia representa la expresión reflexiva subjetiva de lo vivido por el Sujeto, por ende, la Organización de las Naciones Unidas para la Educación, la Ciencia y la 
Cultura (UNESCO, 2012c): revela que tiene valor de conocimiento adquirido por vía de sistematización y organización de competencias demostrable por resultados. Siendo clave la auto reflexión sobre la estructura y aplicación de los resultados.

En conformidad con M'Bow (1987): este tipo de conocimiento implica que el sujeto portador comprenda la subjetividad y configure su mundo socioafectivo-conductual, con la cognición del Ser. Aquí el individuo, al tomar consciencia de lo aprendido en su experiencia, adquiere su propia identidad en el contexto donde se encuentra. Este cuestiona la legitimidad de lo adquirido requiriendo de una mediación institucional para su reconocimiento cognitivo, socioemocional y conductual.

Interpretando a Freire (2011): este conocimiento representaría una construcción sociohistórico y colectivo; en donde el sujeto es capaz de reconocer y apreciar lo adquirido por vía experiencia. Lo que para López (2019): es un conocimiento epistémico práctico facilitador de la interacción social.

\section{Conclusiones}

La presente investigación devela el sentido social que los venezolanos han asignado históricamente al aprendizaje por experiencias. Tanto en el discurso legal, cuyo sentido predominante es de enlace intergeneracional garantizando que sus portadores no sean "cargas sociales", en la medida que sus conocimientos sean actualizados y puedan insertarse en el mundo laboral, ya que en el discurso universitario significa predominante y es una "herramienta flexibilizadora" de la diversidad de protocolos que han representado la universidad en su evolución histórica.

Simbólicamente a nivel social el aprendizaje por experiencia se presenta; al menos, en cinco representaciones sociales aquí denominadas: a). bizarro conocimiento: que vincula el saber popular con el conocimiento 
científico, asociado a enfoques teóricos humanistas; b). demostrativo: aquel conocimiento que requiere ser validado con evidencia para ser considerado por la academia, equiparado al enfoque de aprendizaje deductivo; c). resolutivo: aquel que soluciona problemas concretos y satisface necesidades; y d). por contraste: en donde el sujeto toma conciencia de poseer dicho conocimiento, después de ser sometido a un proceso de comparación.

La develación de estos significados legales y universitarios, al igual que las representaciones sociales, ayudan a comprender por qué el debate y su importancia en el Sistema Educativo Venezolano, a la vez que las dificultades para su reconocimiento, validación y certificación.

\section{Referencias}

Arnaz, J. (2016). La planeación curricular. Segunda edición, ISBN: 9789682438264. México: Editorial Trillas.

Cinterfor/OIT (1990). La formación profesional en el Umbral de los 90: un estudio de los cambios e innovaciones en las instituciones especializadas en América Latina. Volumen 1 y Volumen 2, Primera edición, ISBN: 92-9088-022-7. Montevideo, Uruguay: Organización Internacional del Trabajo.

Delors, J. (1996). Los cuatro pilares de la educación. En La educación encierra un tesoro. Informe a la UNESCO de la Comisión internacional sobre la educación para el siglo XXI. Madrid, España: Santillana/UNESCO. Recuperado de:

https://uom.uib.cat/digitalAssets/221/221918 9.pdf

Dewey, J. (1998). Democracia y Educación: Una introducción a la filosofía de la educación. Tercera edición, ISBN: 84-7112-391-6. Madrid, España: Ediciones Morata, S.L.

Freire, P. (2011). La educación como práctica de la libertad. ISBN: 978607-03-0299-2. España: Siglo XXI Editores, S.A. de C.V. 
Hernández, R., Fernández, C., \& Baptista, L. (2010). Metodología de la Investigación. 5ta Edición, ISBN: 978-607-15-0291-9. México: McGraw-Hill / Interamericana Editores, S.A. de C.V.

Hopenhayn, M., \& Ottone, E. (2000). El gran eslabón: educación y desarrollo en el umbral del siglo XXI. ISBN: 9505573421. Buenos Aires, Argentina: CEPAL, Fondo de Cultura Económica.

López, E. (2019). Preconcepción de la Educación Ambiental a través de las Representaciones Sociales del Docente. Revista Scientific, 4(14), 120-140, e-ISSN: 2542-2987. Recuperado de:

https://doi.org/10.29394/Scientific.issn.2542-2987.2019.4.14.6.120-140 M'Bow, A. (1987). UNESCO y la Educación para todos. Aspectos Cuantitativos y Cualitativos. TyCE: Tecnología y Comunicación Educativas, 3(7), 15-27. México: Instituto Latinoamericano de la Comunicación Educativa, Dirección de Investigación y Contenidos Educativos. Recuperado de:

http://tyce.ilce.edu.mx/tyce/7/TecyComEduNo07 A02.pdf

OIT/Cinterfor (2017). El futuro de la formación profesional en América Latina y el Caribe: diagnóstico y lineamientos para su fortalecimiento. Primera edición, ISBN: 978-92-9088-274-9. Montevideo, Uruguay: Organización Internacional del Trabajo.

Páez, M., \& Puig, J. (2013). La reflexión en el Aprendizaje-Servicio. Riejs: Revista Internacional de Educación para la Justicia Social, 2(2), 13-32, e-ISSN: 2254-3139. Recuperado de:

https://revistas.uam.es/riejs/article/view/370

Strauss, A., \& Corbin J. (2002a,b,c). Bases de la investigación cualitativa. Técnicas y procedimientos para desarrollar la teoría fundamentada. Primera edición, ISBN: 958-655-623-9. Medellín Colombia: Editorial Universidad de Antioquia; Sage Publications, Inc.

Torp, L., \& Sage, A. (2007a,b). El aprendizaje basado en problema: desde 
el jardín de infantes hasta el final de la escuela segundaria. Primera reimpresión, ISBN: 978-950-518-811-6. Madrid, España: Amorrortu Editores España, S.L.

UNESCO (2017). 3er Informe mundial sobre el aprendizaje y la educación de adultos. ISBN: 978-92-820-3290-9. Hamburgo, Alemania: Instituto de la UNESCO para el Aprendizaje a lo Largo de Toda la Vida.

UNESCO (2012a,b,c). Directrices de la UNESCO para el reconocimiento, validación y acreditación de los resultados del aprendizaje no formal e informal. Código del documento: UIL/2012/PI/H/3 REV.2. Hamburgo, Alemania: Instituto de la UNESCO para el Aprendizaje a lo Largo de Toda la Vida.

Valiente B. (2000). Argumentar, demostrar, explicar: ¿continuidad o ruptura cognitiva?. Educación Matemática, 12(2), 149-151, e-ISSN: 2448-8089. México: Sociedad Mexicana de Investigación y Divulgación de la Educación Matemática, A.C.

Verón, E. (2004a,b). Fragmentos de un Tejido. Colección El mamífero parlante: Serie mayor, ISBN: 9788474328738 . Buenos Aires, argentina: Editorial Gedisa.

Verón, E (1993a,b,c). La Semiosis Social. Fragmentos de una teoría de la discursividad. Primera reedición, ISBN: 84-7432-502-1. Barcelona, España: Editorial Gedisa, S.A.

Villiers, M. (1993). El papel y la función de la demostración en matemáticas. Epsilon, (26), 15-30, ISSN: 1131-9321. España: Sociedad Andaluza de Educación Matemática "Thales". 


\section{Maeva Elena Hernández Pereira \\ e-mail: maevaunefa2012@gmail.com}

Nacida en Caracas, Venezuela, el 28 de noviembre del

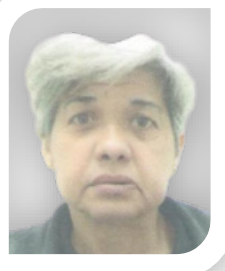
año 1958. Licenciada en Sociología en la Universidad Católica Andrés Bello (UCAB), Caracas, Venezuela; con especialización en Gerencia General, Maestría en Ciencias Administrativa y Doctorado en Ciencias Sociales en la Universidad Central de Venezuela (UCV); y Postdoctorado en Ciencias de la Educación; docente en la Universidad Central de Venezuela; Universidad Nacional Experimental Simón Rodríguez (UNESR); Universidad Nacional Abierta (UNA); y en la Universidad Nacional Experimental Politécnica de la Fuerza Armada (UNEFA), Venezuela.

El contenido de este manuscrito se difunde bajo una Licencia de Creative Commons ReconocimientoNoComercial-Compartirlgual 4.0 Internacional 